\title{
Ab initio description of collectivity for $s d$ shell nuclei
}

\author{
A. Saxena ${ }^{1}$, A. Kumar ${ }^{1}$, V. Kumar ${ }^{2}$, P. C. Srivastava ${ }^{1}$, and T. Suzuki ${ }^{3,4}$ \\ ${ }^{1}$ Department of Physics, Indian Institute of Technology, Roorkee - 247667, INDIA \\ ${ }^{2}$ Department of Physics, Central University of Kashmir, Ganderbal - 190004, INDIA \\ ${ }^{3}$ Department of Physics, College of Humanities and Science, \\ Nihon Univerity, Sakurajosui 3, Setagaya-ku, Tokyo 156-8550, JAPAN and \\ 4 National Astronomical Observatory of Japan, \\ Osawa 2, Mitaka, Tokyo 181-8588, JAPAN
}

\begin{abstract}
In the present work, we have reported shell model results for open shell nuclei $\mathrm{Ne}, \mathrm{Mg}$ and $\mathrm{Si}$ isotopes with $10 \leq N \leq 20$ in $s d$-shell model space. We have performed calculations in $s d$ shell with two ab initio approaches: in-medium similarity renormalization group (IM-SRG) and coupledcluster (CC) theory. We have also performed calculations with phenomenological USDB interaction and chiral effective field theory based CEFT interaction. The results for rotational spectra and $B\left(E 2 ; 2_{1}^{+} \rightarrow 0_{1}^{+}\right)$transitions are reported for even-mass isotopes. The IM-SRG and CC results are in reasonable agreement with the experimental data except at $N=20$. This demonstrates a validity of ab initio description of deformation for doubly open-shell nuclei for $s d$ shell. To see the importance of $p f$ orbitals, we have also compared our results with SDPF-MU interaction by taking account of $2 p-2 h$ and $4 p-4 h$ configurations in $s d-p f$-shell model space.
\end{abstract}

PACS numbers: 21.60.Cs - shell model 


\section{INTRODUCTION}

The structure and properties of nuclei significantly change once we move towards drip-

lines $[1,2]$. The "island of inversion" of nuclei around $\mathrm{A}=30$ has been a subject of several experimental and theoretical studies. There are different portals of island of inversion, for example, they may occurs at $N=8, N=20, N=28, N=40$ and $N=50$. To decide structure of these nuclei, intruder orbitals also become important. Recently there are several experimental groups which involve to study structure of these nuclei, in particular the "island of inversion" region of light nuclei with $N \sim 20$. Thus, from theoretical side apart from naive shell model it is important to study these nuclei with ab initio approaches [3, 4]. As it is now possible to study lower $s d$ shell nuclei using ab initio approaches due to advancement in the computational facility, it is challenging to test predictive power of ab initio calculations for doubly-open shell nuclei for the description of deformation in the medium-mass region.

In the present work our motivation is to test the ab initio Hamiltonians to calculate the spectra and $B(E 2)$ transitions for the doubly-open sd shell nuclei. Previously, we have reported electromagnetic properties, Gamow-Teller (GT) strengths and spectroscopic strengths of $s d$ shell nuclei in Refs. [5-7].

We organize present work as follows. In section 2, we present details about Hamiltonians for ab initio calculations. In section 3, we present theoretical results along with experimental data wherever it is available. Finally summary and conclusions are drawn in section 4.

\section{FORMALISM}

In our studies of neutron-rich $\mathrm{Ne}, \mathrm{Mg}$ and $\mathrm{Si}$ isotopes, we have performed calculations in $s d$ space. As it is well known that excitation to $p f$-shell is very important for nuclei which belong to "island of inversion" [8], thus, we have also performed calculations in sd-pf shell.

For the $s d$ shell, we use the ab initio Hamiltonian derived from two modern ab initio approaches: IM-SRG [3] and CCEI [4, 9]. We have also compared results with a phenomenological USDB effective interaction [10]. For $s d-p f$ shell we have performed calculations with SDPF-MU intercation [11]. For the diagonalization of matrices we have used shell-model code KSHELL [12].

Using IM-SRG approach [13] based on chiral two- and three-nucleon interactions, Stroberg 
et al., derived mass-dependent Hamiltonians for $s d$ shell nuclei [3]. The strategy is that we select a Hamiltonian in certain basis so that the energy states which strongly differ in energy range in off diagonal matrix elements should be eliminated. After applying unitary transformation we get a final Hamiltonian $H(s)$ from a initial Hamiltonian $H(0)$.

$$
H(s)=U^{\dagger}(s) H(0) U(s)=H^{d}(s)+H^{o d}(s) .
$$

Here, $\mathrm{s}$ is the flow parameter. $H^{d}(s)$ is the diagonal part of the Hamiltonian and $H^{\text {od }}(s)$ is the off diagonal part of the Hamiltonian. The flow of the Hamiltonian is obtained by differentiating Eq. (1) w.r.to 's'-

$$
\frac{d H(s)}{d s}=[\eta(s), H(s)]
$$

where $\eta(s)$ is the anti-Hermitian generator of unitary transformation,

$$
\eta(s)=\frac{d U(s)}{d s} U^{\dagger}(s)=-\eta^{\dagger}(s) .
$$

Eq. (2) is known as the flow equation for the Hamiltonian. The off-diagonal matrix elements become zero as $s \rightarrow \infty$ for appropriate value of $\eta(s)$. Here $s d$ valence space decouple from the core and higher shells as $s \rightarrow \infty$. Now we use the resulting Hamiltonian in the shell model calculations with $\hbar \omega=24 \mathrm{MeV}$. Further details about parameters are given in ref. [3].

The Hamiltonian developed from the Coupled Cluster Effective Interaction approach is $A$ - dependent and can be extended as-

$$
H_{C C E I}=H_{0}^{A_{c}}+H_{1}^{A_{c}+1}+H_{2}^{A_{c}+2}+\ldots
$$

Here, $H_{0}^{A_{c}}, H_{1}^{A_{c}+1}$, and $H_{2}^{A_{c}+2}$ are called core, one-body, and two-body cluster Hamiltonians, respectively. This expansion is known as valance cluster expansion. Any operator can be expanded in the valence space in the same way as the Hamiltonian for the shell model calculations. The Okubo-Lee-Suzuki (OLS) similarity transformation is used to calculate the two-body term. In this approach Hartree-Fock ground state in thirteen oscillator major shells with $\hbar \omega=20 \mathrm{MeV}$ is used. Recently, L. Huth et al. [14] derived a shell-model interaction from effective field theory, which will be referred as CEFT. 

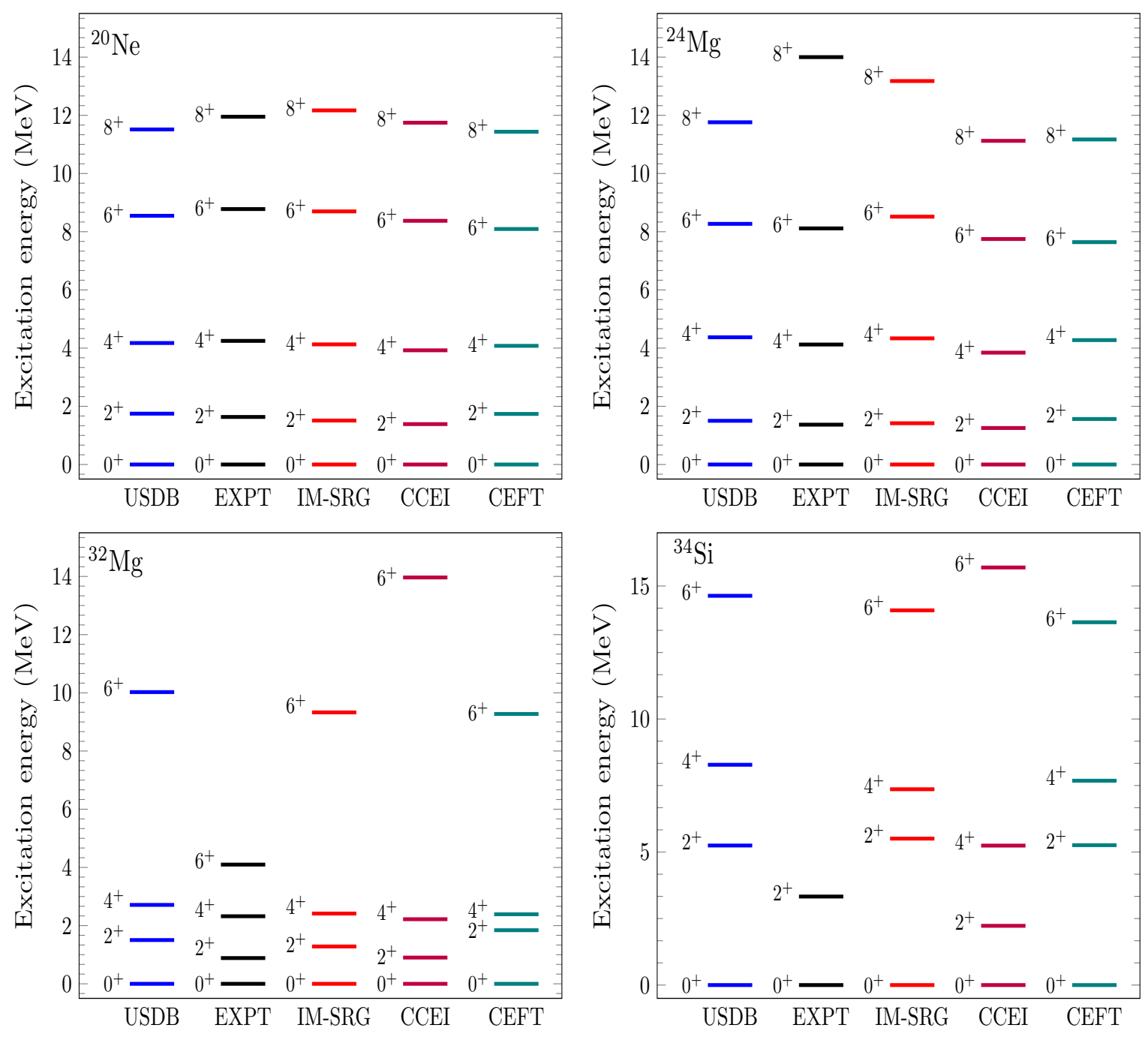

FIG. 1: The energy spectra of ${ }^{20} \mathrm{Ne},{ }^{24} \mathrm{Mg},{ }^{32} \mathrm{Mg}$ and ${ }^{34} \mathrm{Si}$ using USDB, IM-SRG, CCEI and CEFT interactions.

The $B(E 2)$ values are calculated with the formula:

$$
B(E 2)=\frac{1}{2 J_{i}+1}\left|\left(J_{f}\left\|\sum_{i} e_{i} r_{i}^{2} Y_{2}\left(\theta_{i}, \phi_{i}\right)\right\| J_{i}\right)\right|^{2} .
$$

Where, $J_{i}$ and $J_{f}$ are the initial and final state spins, respectively. The $B(E 2)$ values are calculated with the effective charges $e_{p}=1.35 \mathrm{e}$ and $e_{n}=0.35 \mathrm{e}$. 


\section{RESULTS AND DISCUSSIONS}

The experimental measurement of $B(E 2)$ values and nuclear moments are important to predict existence of the "island of inversion". The nuclei lying in the "island of inversion" region show a drastic enhancement of quadrupole collectivity compared to neighboring nuclei from these experimental results.

${ }^{20} \mathrm{Ne}$ provides a good example of rotational spectra [15] in the lower $s d$ shell. The comparison of rotational energy levels for ${ }^{20} \mathrm{Ne}$ obtained by USDB, IM-SRG, CCEI, and CEFT interactions is shown in the Fig. 1. The rotational spectra ${ }^{20} \mathrm{Ne}$ and ${ }^{24} \mathrm{Mg}$ are reproduced in our ab initio calculations. The $E_{2_{1}^{+}}$and $B\left(E 2 ; 2_{1}^{+} \rightarrow 0_{1}^{+}\right)$transitions using ab initio interactions and USDB interactions along with experimental data $[16,17]$ for even Ne isotopes with $N=10-20$ are shown in Fig. 2. The USDB results for $E\left(2_{1}^{+}\right)$are close to experimental data up to $N=16$ but above this results are deviating. The IM-SRG results are the best and close to the experimental data from $N=10$ to $N=18$. At $N=20$, only CCEI shows the same pattern as the experimental data, while results of all the other interactions go upward deviating from the experiment. Experimentally, $N=18$ shows less collectivity in comparison to $N=16$ and an enhancement in collectivity is seen at $N=20$. The $B(E 2)$ values obtained are not satisfactory especially at $N=20$ for $\mathrm{Ne}$ and $\mathrm{Mg}$ isotopes. For all the interactions the collectivity is decreasing from $N=18$ to $N=20$ in contrast to the experiment. From the literature the $N=20$ lies on the boundary of "island of inversion" [18] and $0 \hbar \omega$ shell model calculations are not able to reproduce the enhancement in collectivity at $N=20$.

To see the importance of neutron excitations from $s d$ to $p f$ shell, we have shown results with $2 p-2 h$ and $4 p-4 h$ excitations in Fig. 3. The results with $4 p-4 h$ excitations show the same trends as in the experiment : there is an increase of $B\left(E 2 ; 2_{1}^{+} \rightarrow 0_{1}^{+}\right)$from $N=18$ to $N=20$ though not enough to reproduce the experimental data. The increase in the occupancy of $p f$ orbitals are also confirmed from Fig. 4.

For the $\mathrm{Mg}$ isotopes, the case of ${ }^{32} \mathrm{Mg}$ is very interesting. Its g.s. is deformed and it shows rotational spectrum. Up to $4^{+}$, the CCEI results are very close to the experimental data but

$6^{+}$state is very high in energy. Also, for ${ }^{24} \mathrm{Mg}$, the rotational spectra is well reproduced by $a b$ initio interactions up to $6^{+}$. In Fig. 2, the energy of $2_{1}^{+}$state is near to the experimental data with IM-SRG effective interaction except at $N=14$ and $N=20$. The CCEI results 

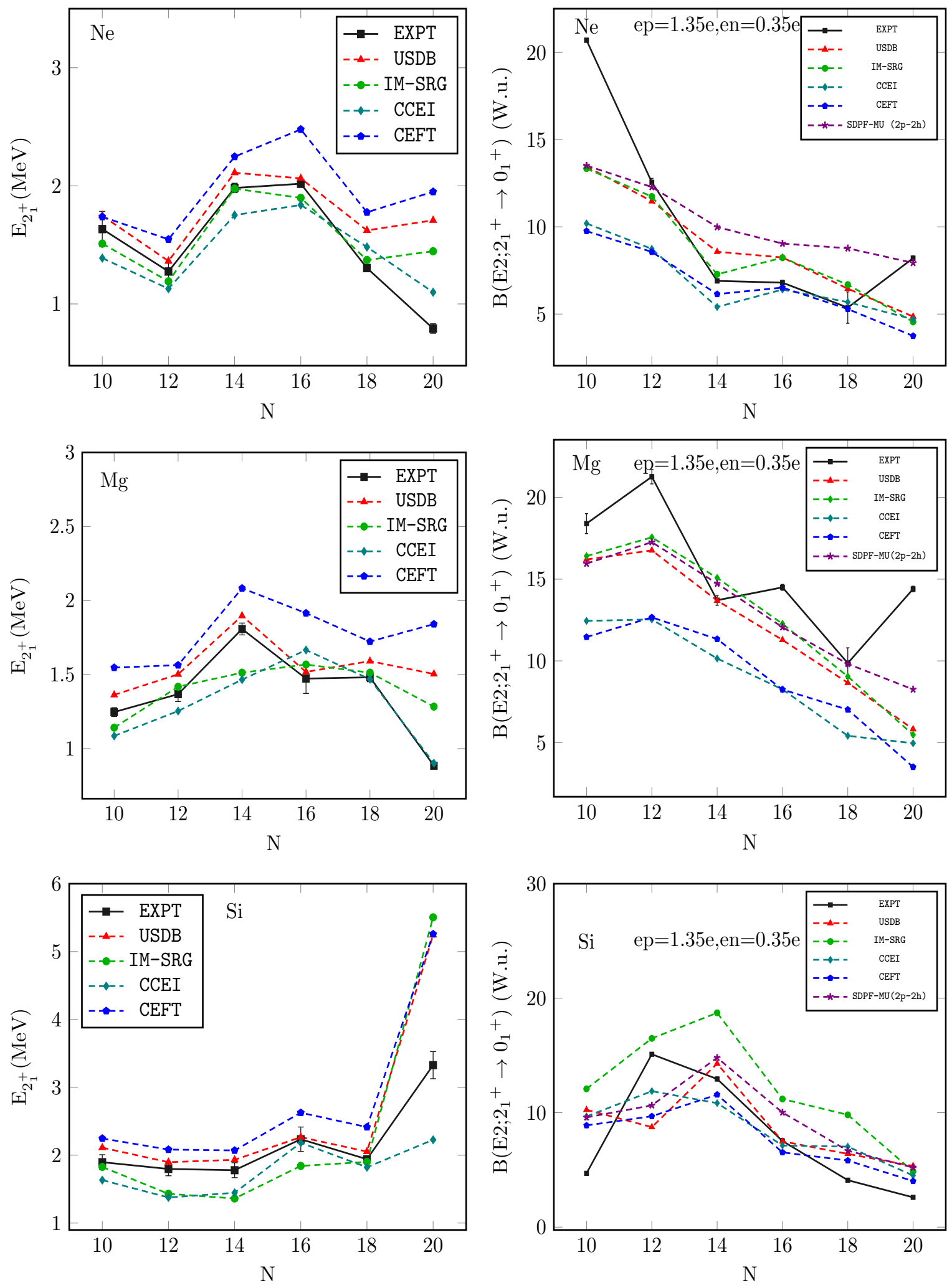

FIG. 2: The energy of $2_{1}^{+}$and $B\left(E 2 ; 2_{1}^{+} \rightarrow 0_{1}^{+}\right)$values of $\mathrm{Ne}, \mathrm{Mg}$ and Si isotopes. 

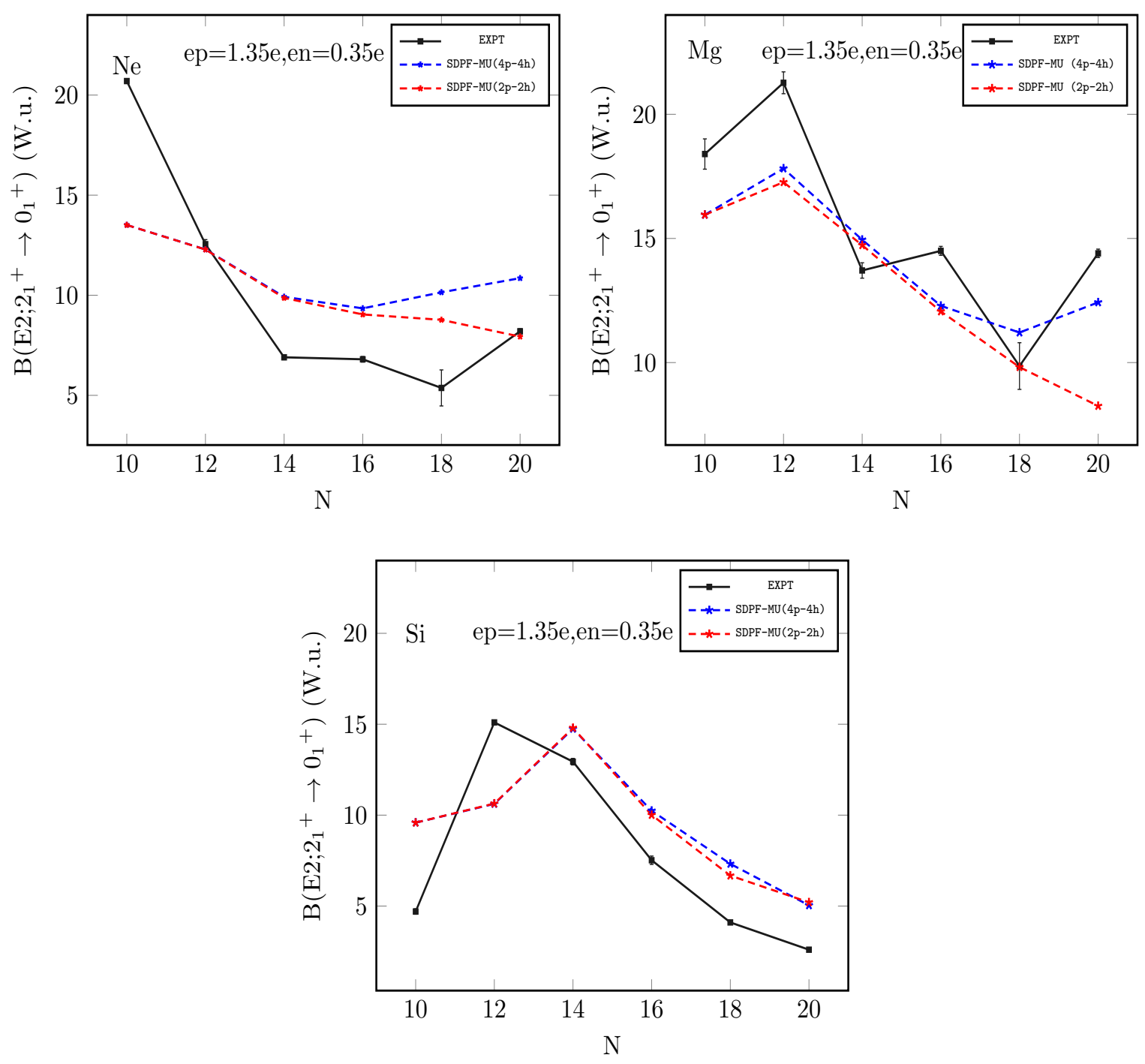

FIG. 3: Comparison between calculated and experimental $B\left(E 2 ; 2_{1}^{+} \rightarrow 0_{1}^{+}\right)$values of $\mathrm{Ne}, \mathrm{Mg}$ and Si isotopes for $s d-p f$ shell with $2 p-2 h$ and $4 p-4 h$ excitations.

are also good except at $N=14$. At $N=20$ the energy of $2_{1}^{+}$state is correctly given by CCEI but $B(E 2)$ value is far from the experimental data. This shows there is a problem with the wavefunction, that is, there is a large configuration mixing in this wavefunction. Here, with ab initio interactions it is not possible to show collectivity at $N=20$. The shell model results with $2 p-2 h$ and $4 p-4 h$ exciations for SDPF-MU interaction are shown in Fig. 3. They show smooth decrease in $B(E 2)$ values from $N=12-16$ for both $2 p-2 h$ and $4 p-4 h$ excitations, however, the results of $4 p-4 h$ show the same trends as in the experiment at $N=20$ : there is an increase of $B\left(E 2 ; 2_{1}^{+} \rightarrow 0_{1}^{+}\right)$from $N=18$ to $N=20$. 

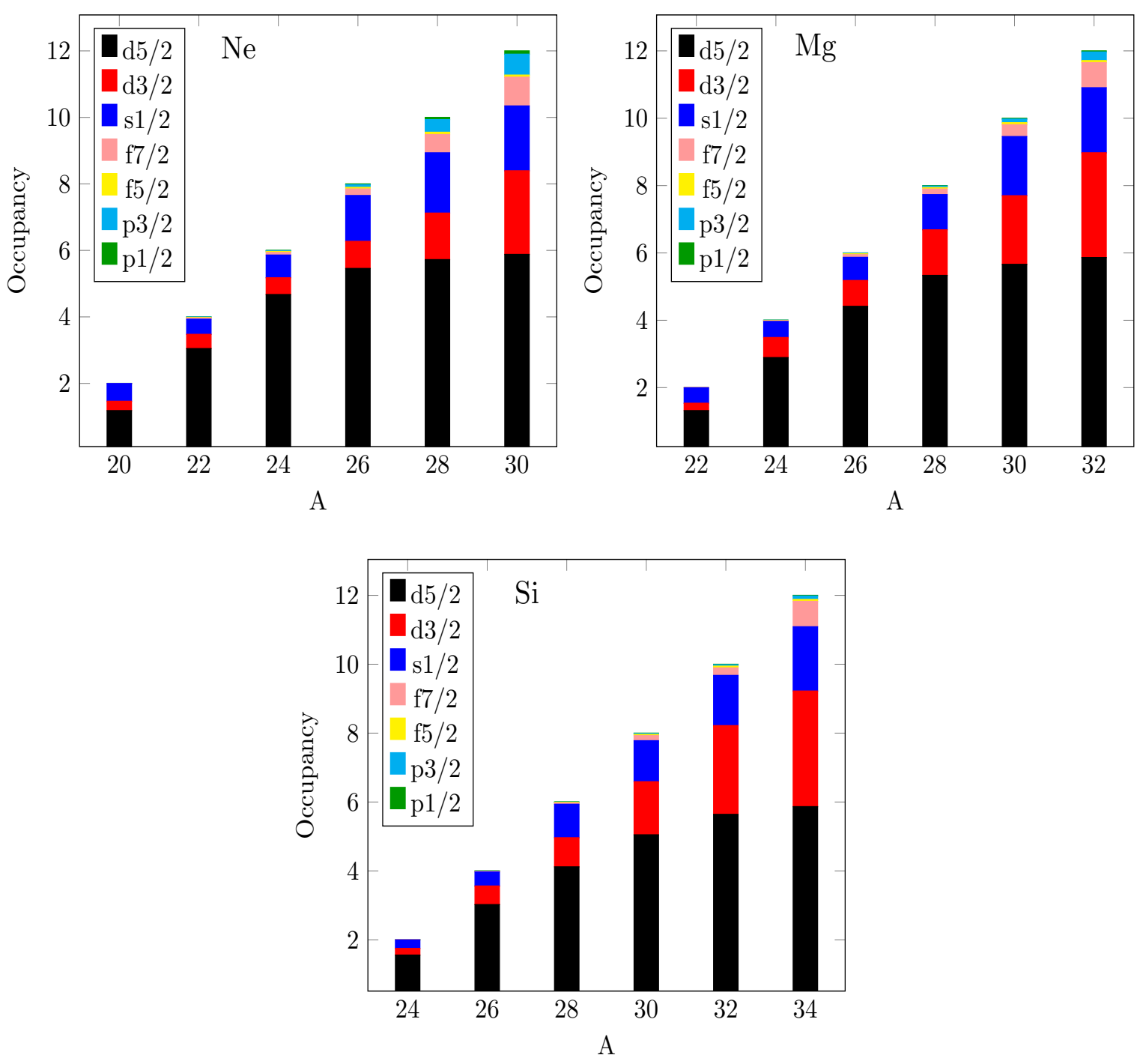

FIG. 4: The neutron occupancy of $2_{1}^{+}$state for $\mathrm{Ne}, \mathrm{Mg}$ and $\mathrm{Si}$ isotopes with SDPF-MU interaction with $2 p-2 h$ excitations .

In the case of ${ }^{34} \mathrm{Si}$ isotope none of the interaction explain properly the spectra. For $\mathrm{Si}$ isotopes the trend of energy of $2_{1}^{+}$state for $N=10$ to $N=18$ isotopes are well predicted by CEFT and USDB interactions. The $B\left(E 2 ; 2_{1}^{+} \rightarrow 0_{1}^{+}\right)$trend from $N=12$ to $N=18$ are showing reasonable agreement with the experiment for all the interactions. The $B\left(E 2 ; 2_{1}^{+} \rightarrow\right.$ $0_{1}^{+}$) values for the case with $2 p-2 h$ and $4 p-4 h$ excitations show similar results.

In the present calculations the gap between $d_{3 / 2}$ orbital and $f p$ shell is large for the SDPF-MU interaction compared with the interaction in Ref. [1], where the neutron ESPE's of $p f$-shell are very close to those $s d$-shell; the difference between $f_{7 / 2}$ and $d_{3 / 2}$ is as small as 
about $2 \mathrm{MeV}$ for $\mathrm{Z}=12(\mathrm{~N}=20)$. Therefore, even if we allow $4 p-4 h$ excitation from $s d$ to $p f$ shell, the occupancy of $f p$-shell are around 2.23 in ${ }^{32} \mathrm{Mg}$. As we can see in Ref. [1], where energies and $B(E 2)$ are well reproduced up to $N=20$, the occupancy of $f p$-shell becomes as large as 3.5 for ${ }^{32} \mathrm{Mg}$.

Thus our results might be improved if we reduce the gap between $s d$ and $f p$ shells for the SPDF-MU interaction.

\section{SUMMARY AND CONCLUSIONS}

In the present work, we have performed shell model calculations for open shell nuclei with $10 \leq N \leq 20$ for $\mathrm{Ne}, \mathrm{Mg}$ and $\mathrm{Si}$ isotopes in the $s d$ and $s d-p f$ spaces. For $s d$ shell we have taken two ab initio interactions, IM-SRG and CCEI. We have also performed calculations with phenomenological USDB and interaction based on chiral effective field theory. The degree of freedom of $s d$ and $p f$ shells are essential for the nuclei close to "island of inversion" so, we have also reported results of $s d-p f$ shell with SDPF-MU interaction. The results of $a b$ initio interactions show reasonable agreement with the experimental data except at $N$ $=20$. For nuclei in the island of inversion such as ${ }^{30} \mathrm{Ne}$ and ${ }^{32} \mathrm{Mg}$, the admixture of $p f$ shells

is important to explain the lowering of the energies of $2_{1}^{+}$states and the enhancement of the $B(E 2)$ values. present study will add more information to earlier theoretical $B(E 2)$ values of $\mathrm{Ne}, \mathrm{Mg}$ and $\mathrm{Si}$ isotopes.

\section{Acknowledgments}

AS acknowledges financial support from MHRD (Govt. of India) for her Ph.D. thesis work. Vikas Kumar acknowledges partial support from CUK.

[1] N. Tsunoda, T. Otsuka, N. Shimizu, M. Hjorth-Jensen, K. Takayanai, and T. Suzuki, Phys. Rev. C 95, 021304(R) (2017).

[2] H. L. Crawford, P. Fallon, A. O. Macchiavelli, A. Poves, V. M. Bader, D. Bazin, M. Bowry, C. M. Campbell, M. P. Carpenter, R.M. Clark et al., Phys. Rev. C 93, 031303(R) (2016). 
[3] S. R. Stroberg, H. Hergert, J. D. Holt, S. K. Bogner, and A. Schwenk, Phys. Rev. C 93, 051301(R) (2016).

[4] G. R. Jansen, J. Engel, G. Hagen, P. Navratil, and A. Signoracci, Phys. Rev. Lett. 113, 142502 (2014).

[5] A. Saxena and P.C. Srivastava, Phys. Rev. C 96, 024316 (2017).

[6] A. Saxena, P.C. Srivastava and T. Suzuki, Phys. Rev. C 97, 024310 (2018).

[7] P.C. Srivastava and V. Kumar, Phys. Rev. C 94, 064306 (2016).

[8] A. O. Macchiavelli et al., Phys. Rev. C 94, 051303(R) (2016).

[9] G. R. Jansen, M. D. Schuster, A. Signoracci, G. Hagen, and P. Navrátil, Phys. Rev. C 94, 011301(R) (2016).

[10] B. A. Brown and W. A. Richter, Phys. Rev. C 74, 034315 (2006).

[11] Y. Utsuno, T. Otsuka, B.A. Brown, M. Honma, T. Mizusaki and N. Shimizu, Phys. Rev. C 86, 051301(R) (2012).

[12] N. Shimizu, "Nuclear shell-model code for massive parallel computation, KSHELL" (private communication).

[13] S.K. Bogner, R.J. Furnstahl and A. Schwenk, Prog. Part. Nucl. Phys. 65, 94 (2010).

[14] L. Huth, V. Durant, J. Simonis, ans A. Schwenk, Phys. Rev. C 98, 044301 (2018).

[15] Fundamentals of nuclear models, D.J. Rowe and J.L. Wood, World Scientific (2010).

[16] Data extracted using the NNDC World Wide Web site from the ENSDF database.

[17] B. Pritychenko, M. Birch, B. Singh, and M. Horoi, At. Data and Nucl. Data Tables 107, 1 (2016).

[18] H. Iwasaki et al., Phys. Lett. B 620, 118 (2005). 